\section{Prescripción potencialmente inadecuada en patología cardiovascular en pacientes ancianos en atención primaria}

\section{Potentially inappropriate prescription in cardiovascular pathology in elderly patients in primary care}

Néstor J. Sánchez Sánchez'

Mercedes A. Hernández Gómez

Ramón Otero

Adalid Maldonado Díaz ${ }^{1}$

Elvira Santiago Janeiro²

Alejandra Martínez Franco ${ }^{1}$

1. Médico de familia. Centro de Salud Novoa Santos. Ourense. España

2. Enfermero. Centro de Salud Novoa Santos. Ourense. España.

*Autor para correspondencia.

Correo electrónico: njsanchezs@gmail.com (Néstor J. Sánchez Sánchez).

Recibido el 2 de diciembre de 2019; aceptado el 11 de febrero de 2020.

\section{RESUMEN}

Objetivo: Estudiar en pacientes mayores de 65 años las prescripciones potencialmente inapropiadas y omisiones de prescripción en patología cardiovascular en atención primaria. Metodología: Estudio descriptivo transversal en un centro de salud urbano utilizando la versión 2 de los criterios STOPP-START. Resultados: En los 170 pacientes incluidos

(62,4\% mujeres; mediana de edad, 79 años) hubo un 15,3\% de prescripciones potencialmente inapropiadas y un $8,8 \%$ de omisiones de prescripción. El 72,9\% tenía más de 4 enfermedades y el 57,1\% tomaba más de 4 fármacos. A mayor pluripatología, mayor consumo de fármacos, sin relación entre las prescripciones potencialmente inapropiadas y las características epidemiológicas y clínicas de los pacientes. Conclusiones: Los criterios STOPP-START son útiles para revisar la prescripción en personas mayores de 65 años con enfermedades cardiovasculares entre su pluripatología.

PALABRAS CLAVE: Polifarmacia, prescripción inapropiada, omisión de la prescripción, anciano, atención primaria.

\section{ABSTRACT}

Objective: To study potentially inappropriate prescriptions and prescription omissions in cardiovascular pathology in primary care in patients older than 65 years old. Methodology: Descriptive cross-sectional study in an urban primary health center using version 2 of STOPP-START criteria. Results: 170 patients included, $62.4 \%$ women, overage 79 years old, there were $15.3 \%$ potentially inappropriate prescriptions and $8.8 \%$ prescription omissions. $72.9 \%$ had more than 4 diseases and $57.1 \%$ took more than 4 drugs. The more diseases, the more consumption of drugs, without relation between potentially inappropriate prescriptions and the epidemiological and clinical characteristics of the patients. Conclusions: STOPP-START criteria are useful for reviewing prescription in people over 65 with cardiovascular disease among their pluripatology.

KEYWORDS: Polypharmacy, inappropriate prescription, omission prescription, elderly, primary care.

\section{- INTRODUCCIÓN}

La prescripción de múltiples medicamentos por enfermedad multiorgánica aumenta la probabilidad de efectos adversos e interacciones farmacológicas; los errores en la prescripción por exceso [prescripción potencialmente inapropiada (PPI)] o por defecto [omisión de prescripción (OP)], las interacciones farmacológicas y la prescripción igual o superior a 10 fármacos es un indicador de mortalidad en pacientes mayores ${ }^{1-3}$.

Entre las herramientas diseñadas para su detección están los criterios STOPP/START (Screening Tool of Older Persons potentially inappropriate Prescriptions/Screening Tool to Alert doctors to the Right Treatment) $)^{1,4}$. Constan de 65 criterios STOPP para detección de PPI y 22 START para OP en presencia de ciertas enfermedades, salvo contraindicación ${ }^{5}$. Son aplicables a personas de 65 años o más y existe la versión validada en español ${ }^{1}$ y otra actualizada a nuevas evidencias y fármacos ${ }^{6}$. Su uso ha mostrado efectividad en la práctica clínica ${ }^{7}$; sin embargo, son escasos los estudios realizados exclusivamente en atención primaria frente a los realizados en hospitales o residencias geriátricas ${ }^{3,4,8-10}$.

Las enfermedades cardiovasculares constituyen la primera causa de mortalidad en la población espańola y engloba la mayor parte de la prescripción en ancianos polimedicados ${ }^{6,10,11}$. $\mathrm{Al}$ alta hospitalaria supone $\geq$ 1 PPI y mayor riesgo de presentar algún evento adverso, y la OP se asocia con reingreso ${ }^{12}$.

\section{- OBJETIVOS}

Principal: identificar las PPI y OP mediante criterios STOPP/START en un centro de salud urbano.

Secundarios: identificar los factores asociados en personas con enfermedad cardiovascular mayores de 65 ańos; identificar las PPI y OP en función de las características demográficas. 


\section{- MÉTODOS}

Se trata de un estudio descriptivo transversal. La población incluye pacientes de 65 o más años con enfermedad cardiovascular atendidos en centro de salud urbano docente de Espańa que acudieron dos o más veces durante el período de estudio. Tener enfermedad cardiovascular incluyó cardiopatía isquémica, insuficiencia cardíaca, enfermedad cerebrovascular, arteriopatía periférica y enfermedad tromboembólica. Fueron excluidos pacientes terminales y pertenecientes a otros cupos.

El cálculo del tamaño muestral se realizó basándose en la prevalencia estimada de PPI en estudios previos realizados en población similar con la misma herramienta ${ }^{3}$. Para una prevalencia esperada de PPI del 20\%, precisión del $5 \%$ y confianza del $95 \%$, se precisaron 169 sujetos. Fueron captados del listado de agenda mediante aleatorización simple. El periodo de estudio fue de junio a octubre de 2016. Los pacientes fueron reclutados al azar conforme acudieron a consulta durante los 5 meses que duró la captación; para ser incluidos debían vivir en la comunidad y dar su consentimiento para participar.

Variables independientes: edad, sexo, número de fármacos crónicos, comorbilidad. Para el análisis de datos se crearon polimedicación (cuatro o más fármacos crónicos), pluripatología (cuatro o más enfermedades crónicas) y edad agrupada en 65-75 años, 75-85 años y más de 85 años.

Variables dependientes: PPI identificada mediante los 9 criterios de la versión 2 de STOPP y las 4 OP de START. Criterios STOPP considerados para detección de PPI:

1. Diuréticos de asa para los edemas maleolares aislados sin signos clínicos de insuficiencia cardíaca.

2. Diuréticos de asa como monoterapia de primera línea en la hipertensión arterial (HTA).

3. Diuréticos tiazídicos con antecedente de gota.

4. Betabloqueantes no cardioselectivos en enfermedad pulmonar obstructiva crónica (EPOC).

5. Asociación del uso de antagonistas del calcio y estreñimiento crónico.

6. Dipiridamol como monoterapia en prevención cardiovascular secundaria.

7. Ácido acetilsalicílico (AAS) en dosis superior a $150 \mathrm{mg} /$ día.

8. AAS sin antecedente de cardiopatía isquémica, enfermedad cerebrovascular, arterial periférica o antecedente oclusivo arterial.

9. Acenocumarol durante más de 12 meses en primera embolia de pulmón no complicada.

Los criterios START considerados OP:

1. No AAS/clopidogrel/estatina en arterioesclerosis.

2. No inhibidor de la enzima de conversión de la angiotensina (IECA) o ARAII en insuficiencia cardíaca congestiva (ICC).

3. No IECA/ARAII en antecedente de infarto agudo de miocardio (IAM). 4. No betabloqueante en ángor.

Los datos se recogieron de historia clínica electrónica-IANUS por el grupo investigador constituido por médicos de familia, enfermería y residentes de atención primaria.

El estudio cumple la Declaración de Helsinki de la World Medical Association (WMA) y las recomendaciones de la European Medicines Agency (EMA). Código de Registro del Comité de Ética de la Investigación de Pontevedra-Vigo-Ourense: 2016/572.

La presente investigación no ha recibido ayudas específicas provenientes de agencias del sector público, sector comercial o entidades sin ánimo de lucro.

Los datos se recogieron a partir de historia clínica-IANUS en un cuaderno de recogida de datos por paciente.
El análisis de datos se hizo mediante estadística descriptiva: las variables cuantitativas se describieron como mediana, mínimo y máximo en distribuciones asimétricas. Las variables cualitativas se describieron mediante frecuencias y porcentaje. Se compararon las variables demográficas y polimedicación utilizando pruebas paramétricas/no paramétricas ( $\chi^{2}$, Fisher, U de Mann-Whitney).

Los factores asociados a PPI y a OP se estudiaron con modelos de regresión logística univariante y multivariante. Se consideraron estadísticamente significativos los valores de $p<0,05$. Para el análisis de los datos se utilizó el software SPSS 22.0.

\section{- RESULTADOS}

Se incluyeron 170 pacientes; un 62,4\% mujeres. Mediana de edad de la muestra: 79 años (mínimo 65, máximo 100), la mayoría en el rango de edad comprendido entre 75 y 85 años. El 72,9\% presentó pluripatología y el 57,1\%, polifarmacia (más de 4 fármacos).

La tabla 1 muestra los criterios STOPP y START en el área cardiovascular. La PPI afectó a 26 pacientes $(15,3 \%)$ y la OP a 15 (8,8\%).

No se encontró relación significativa entre PPI y sexo, salvo en prescripción de diuréticos en pacientes con hiperuricemia, ya que todos eran varones $(p<0,001)$. Por tramos de edad existió relación entre diurético de ASA y HTA en muy ancianos $(p=0,002) ; 5$ de los 7 pacientes tenían más de 85 ańos.

De los 94 pacientes con arterioesclerosis, el 55,3\% tomó AAS/clopidogrel; el 78,7\%, estatina; y el 34,04\%, AAS/clopidogrel y estatina simultáneamente. En ningún caso de arterioesclerosis se tuvo que comenzar a dar medicación para dicha patología.

Tabla 1. Descripción de la prescripción potencialmente inapropiada según criterios STOPP y de omisión de prescripción según criterios START en pacientes con enfermedad cardiovascular mayores de 65 años

Frecuencia Porcentaje (\%)

PPI cardiovascular según criterios STOPP

\begin{tabular}{|l|l|l|}
\hline Tiazídicos en gota & 8 & 4,70 \\
\hline Diurético de asa por HTA & 7 & 4,10 \\
\hline AAS >150 & 5 & 2,90 \\
\hline Antagonista del calcio y estreñimiento & 3 & 1,80 \\
\hline Diurético de asa por edema & 1 & 0,60 \\
\hline Betabloqueante en EPOC & 1 & 0,60 \\
\hline AAS sin antecedentes personales & 1 & 0,60 \\
\hline Dipiridamol en prevención secundaria & 0 & 0 \\
\hline Sintrom>12 meses tras TEP & 0 & 0 \\
\hline OP cardiovascular según criterios START & & \\
\hline No AAS/clopidogrel/estatina en & 0 & 0 \\
\hline arterioesclerosis & 4 & 2,4 \\
\hline No tratamiento IECA/ARA Il en ICC & 6 & 3,5 \\
\hline No tratamiento con IECA/ARA Il en IAM & 6 & 3,5 \\
\hline No tratamiento con betabloqueante en & & \\
\hline ángor & &
\end{tabular}

AAS: ácido acetilsalicílico; HTA: hipertensión arterial; IAM: infarto agudo miocardio ICC: insuficiencia cardíaca congestiva; IECA: inhibidores de la enzima de conversión de la angiotensina; TEP: tromboembolismo pulmonar. 
Tabla 2. Relación entre la edad de los pacientes, agrupada por tramos, y la pluripatología. Tabulación cruzada

\begin{tabular}{|l|l|c|c|c|c|}
\hline \multirow{2}{*}{ Pluripatología } & \multicolumn{4}{|c|}{ Edad por tramos } \\
\cline { 2 - 6 } & & $\begin{array}{c}65-74 \\
\text { años }\end{array}$ & $\begin{array}{c}75-85 \\
\text { años }\end{array}$ & $\begin{array}{c}>85 \\
\text { años }\end{array}$ & Total \\
\hline \multirow{2}{*}{$\leq 4$ enfermedades } & Frecuencia & 23 & 12 & 11 & 46 \\
\hline & $\%$ & $35,9 \%$ & $16,9 \%$ & $31,4 \%$ & $27,1 \%$ \\
\hline \multirow{2}{*}{$>4$ enfermedades } & Frecuencia & 41 & 59 & 24 & 124 \\
\hline Total & $\%$ & $64,1 \%$ & $83,1 \%$ & $68,6 \%$ & $72,9 \%$ \\
\hline & Frecuencia & 64 & 71 & 35 & 170 \\
\hline
\end{tabular}

Tabla 3. Relación entre polimedicación y polipatología. Tabulación cruzada

\begin{tabular}{|l|c|c|c|c|}
\hline \multirow{2}{*}{ Polimedicación } & \multicolumn{3}{|c|}{ Polipatología } \\
\cline { 3 - 5 } & & \multicolumn{3}{|c|}{$\leq 4$} \\
enfermedades & enfermedades & Total \\
\hline$\leq 4$ fármacos & Frecuencia & $34(73,9 \%)$ & $39(31,5 \%)$ & $73(42,9 \%)$ \\
\hline$>4$ fármacos & Frecuencia & $12(26,1 \%)$ & $85(68,5 \%)$ & $97(57,1 \%)$ \\
\hline Total & Frecuencia & 46 & 124 & 170 \\
\hline
\end{tabular}

En el análisis bivariado mediante $\chi^{2}$ para OP y sexo se encontró asociación entre OP y ser paciente varón: 10 de las 15 OP en estos pacientes $(p=0,015)$. Por patología, 4 pacientes varones $(21,1 \%)$ presentaron insuficiencia cardíaca congestiva (ICC) y ninguno estaba tratado con IECA/ARA II, encontrándose asociación significativa $(p=0,030)$. En el resto de OP no hubo diferencias significativas en función del sexo, ni relación entre omisión de fármaco y grupo de edad.

Los pacientes con mayor frecuencia de pluripatología pertenecieron al grupo de 75 a 85 ańos $(p=0,037)$, también los de mayor polimedicación $(p=0,023)$ (tabla 2).

No se encontró relación entre polimedicación y pluripatología en función del sexo ( $p=0,265$ y $p=0,639$, respectivamente); sí entre polimedicación y pluripatología $(p<0,001)$; a mayor pluripatología, mayor número de medicamentos prescritos (tabla 3 ).

En el análisis mediante regresión logística entre PPI con las características epidemiológicas y clínicas de los pacientes no se encontró asociación significativa con sexo $(p=0,158)$, edad $(p=0,856)$, pluripatología $(p=0,235)$ o polimedicación $(p=0,351)$.

\section{- DISCUSIÓN}

La prevalencia de PPI en el área cardiovascular fue del 15\% y la de OP del $8,8 \%$. Estas cifras en PPI son similares a las del estudio de Sotoca realizado en ingresados en residencias y en OP inferiores al mismo ${ }^{4}$.
En este trabajo, al igual que en el nuestro, aparecen como principales PPI detectadas mediante criterios STOPP el uso de diurético tiazídico en pacientes con antecedente de hiperuricemia y el uso de diurético de ASA para tratar edemas y HTA en pacientes muy ancianos ${ }^{4}$. En otros son la prescripción de AAS sin indicación clara y el uso de antagonistas del calcio en personas con estreńimiento ${ }^{3}$.

Entre las escasas OP detectadas mediante criterios START destacaron la ausencia de tratamiento con IECA en IAM y/o de betabloqueante en pacientes con ángor, frente a la OP de uso de estatinas frecuente en otros trabajos ${ }^{3,9}$.

En estos estudios, al igual que en el nuestro, la incidencia de criterios STOPP es superior a la de START, siendo así la PPI uno de los principales tipos de errores de medicación identificados en nuestro medio ${ }^{4}$.

Se confirma la asociación entre polimedicación y pluripatología $3,6,13,14$; en nuestro caso, más frecuente en el tramo de edad de 75 a 85 años. En el trabajo de Parodi, PPI y OP se asocian a polimedicación ${ }^{3}$, pero nosotros no encontramos factores predictivos de PPI en función del sexo del paciente, edad, pluripatología o polimedicación.

El presente estudio presenta algunas limitaciones inherentes al diseño y al ámbito de estudio que limitan su potencia. El tamańo muestral y el ámbito de estudio limitan la generalización de los datos, aunque puede servir de base para otros estudios y conocer la prevalencia de PPI y OP en patología cardiovascular en personas mayores en atención primaria mediante criterios STOPP/START. También hubiera sido interesante una valoración de la situación funcional y sociofamiliar de los pacientes o de variables del profesional. La inadecuada cumplimentación de algunas historias ha supuesto otra limitación; algunos criterios STOPP detectados se deben a tratamientos prescritos y no documentados. En el caso de OP sucede algo similar y no se registra la negativa del paciente a tomar la medicación.

Los bajos porcentajes de PPI y de OP detectados en este y otros trabajos $^{3}$ pueden ser asumibles en el proceso de revisión de prescripción de medicamentos. Se necesita investigación adicional para profundizar en estas herramientas, especialmente en ancianos frágiles y pacientes que viven en la comunidad ${ }^{15}$, por lo que este estudio se ajusta a las recomendaciones dadas por otros autores ${ }^{3,15}$

En pacientes ancianos, más vulnerables a problemas relacionados con la medicación, la revisión sistemática de la prescripción es una herramienta útil para detectar PPI y OP. Nuestro estudio muestra que la revisión de prescripción en historia clínica por los profesionales sanitarios aplicando criterios STOPP/START puede mejorar la calidad de la misma y la relación entre profesionales sanitarios de la unidad de asistencia básica.

Por todo ello, sacamos las siguientes conclusiones:

- Los datos obtenidos son similares a los estudios realizados en ámbito hospitalario (PPI 15\%; OP 8,6\%); siendo superior la incidencia de criterios STOPP que la de START.

- No encontramos relación de PPI con la edad, sexo, pluripatología ni polimedicación

\section{Conflicto de intereses}

Todos los autores declaramos no tener conflictos de intereses.

\section{BIBLIOGRAFÍA}

\footnotetext{
1. Delgado Silveira E, Muñoz García M, Montero Errasquin B, Sánchez Castellano C, Gallagher PF, Cruz-Jentoft AJ. Prescripción inapropiada de medicamentos en los pacientes mayores: Ios criterios STOPP/START. Rev Esp Geriatr Gerontol. 2009;44(5):273-79. https://doi.org/10.1016/j.regg.2009.03.017
}

2. Rivas-Cobas PC, Ramírez-Duque N, Gómez Hernández M, García J, Agustí $A$, Vidal $X$, et al. Characteristics of potentially inappropriate prescribing of drugs in elderly polypathological patients. Gac Sanit. 2017:31:327-31. https://doi.org/10.1016/j.gaceta.2016.06.013
3. Parodi López N, Villán Villán YF, Granados Menéndez MI, Royuela A. Prescripción potencialmente inapropiada en mayores de 65 años en un centro de salud de atención primaria. Aten Primaria. 2014:46(6):290-7. https://doi.org/10.1016/j.aprim.2013.12.007 
4. Sotoca Momblona JM, Anglada Martínez H, Molas Ferrer G, Fontanals Martínez S, Rovira lllamola M, Sebastián Montal L. Aplicación de los nuevos criterios de prescripción inadecuada STOPP-START a pacientes geriátricos institucionalizados. FAP. 2011:9(1):2-7. https://doi.org/10.1016/S2172-3761(11)70012-7

5. Gallagher $P$, Baeyens JP, Topinkova E, Madlova P, Cherubini A Gasperini B, et al. Inter-rater reliability of STOPP (Screening Too of Older Persons' Prescriptions) and START (Screening Tool to Alert doctors to Right Treatment) criteria amongst physicians in six European countries. Age Ageing. 2009;38(5):603-6. https://doi org/10.1093/ageing/afp058

6. Blanco-Reina E, García-Merino MR, Ocaña-Riola R, Aguilar-Cano L, Valdellós J, Bellido-Estevez I, et al. Assessing Potentially Inappropriate Prescribing in Community-Dwelling Older Patients Using the Updated Version of STOPP-START Criteria: A Comparison of Profiles and Prevalences with Respect to the Original Version. PLOS ONE. 2016;11(12):e0167586. https://doi.org/10.1371/journal.pone. 0167586

7. Hill-Taylor B, Walsh KA, Stewart S, Hayden J, Byrne S, Sketris IS. Effectiveness of the STOPP/START (Screening Tool of Older Persons' potentially inappropriate Prescriptions/Screening Tool to
Alert doctors to the Right Treatment) criteria: systematic review and meta-analysis of randomized controlled studies. J Clin Pharm Ther. 2016;41(2):158-69. https://doi.org/10.1111/jcpt.12372

8. Castillo-Páramo A, Pardo-Lopo R, Gómez-Serranillos IR, Verdejo A Figueiras A, Clavería A. Valoración de la idoneidad de los criterios STOPP/START en el ámbito de atención primaria en España por el método RAND. Semergen. 2013;39(8):413-20. https://do org/10.1016/.j.semerg.2013.01.017

9. Candela Marroquín E, Mateos Iglesia N. Palomo Cobos L. Adequacy of medication in patients 65 years or older in teaching health centers in Caceres, Spain. Rev Esp Salud Pública. 2012;86(4):41934. doi: 10.4321/S1135-57272012000400009

10. Sevilla-Sánchez D, Espaulella-Panicot J, de Andrés-Lazaro AM, Torres-Allezpuz R, Soldevila-Llagostera M, Codina-Jane C. Medicación potencialmente inapropiada al ingreso en una unidad de media estancia según los criterios STOPP \& START. Rev Esp Geriatr Gerontol. 2012;47(4):155-57. https://doi.org/10.1016/j.regg.2012.02.013

11. Primejdie DP, Traian Bojita M, Popa A. Potentially inappropriate medications in elderly ambulatory and institutionalized patients: an observational study. BMC Pharmacol Toxicol. 2016:17(1):38. https://doi.org/10.1186/s40360-016-0081-x
12. Rodríguez del Río E, Perdigones J, Fuentes Ferrer M, González del Castillo J, González Armengol J Borrego Hernando Ml, et al. Impacto de los resultados a medio plazo de la prescripción inadecuada en los pacientes ancianos dados de alta desde una unidad de corta estancia. Aten Primaria. 2017. https://doi.org/10.1016/j. aprim.2017.03.018

13. Campins LI, Serra-Prat M, Palomera E, Bolibar I, Martínez MA Gallo P. Reduction of pharmaceutical expenditure by a drugs appropiateness intervention in polymedicated elderly subjects in Catalonia (Spain). Gac Sanit. 2017. https://doi.org/10.1016/j. gaceta.2017.09.002

14. Garfinkel D. Mangin D. Feasibility study of a systematic approach for discontinuation of multiple medications in older adults: addressing polypharmacy. Arch intern Med. 2010;170(18):1648-54 doi:10.1001/archinternmed.2010.355

15. Hill-Taylor B. Sketris I, Hayden J, Byrne S, O'Sullivan D, Christie R. Application of the STOPP/START criteria: a systematic review of the prevalence of potentially inappropriate prescribing in older adults, and evidence of clinical, humanistic and economic impact. J Clin Pharm Ther. 2013;38(5):360-72. https://doi. org/10.1111/jcpt.1205 